\title{
Application of Computer Technology in Sports Training and Competition
}

\author{
Changgui $\mathrm{He}^{1}$, Chao $\mathrm{Ye}^{2}$ \\ ${ }^{1}$ Polytechnic Institute of Jiangxi Science \& Technology Normal University, Nanchang Jiangxi \\ 330100, China; \\ ${ }^{2}$ Polytechnic Institute of Jiangxi Science \& Technology Normal University, Nanchang Jiangxi \\ 330100, China \\ 346591653@163.com
}

KEYWORDS: Sports Training, Sports Competition, Computer Technology, Application Analysis

\begin{abstract}
With the continuous development of information technology, computer technology began gradually applied to sports training and sports were to go, so as to achieve better practical results. The application of computer technology to the modern sports training and sports, you can accurately obtain the corresponding results of the competition, provided the corresponding data for the reference to training coaches reasonable adjustments. This article is mainly for computer technology in sports training and sports were exploration and research, in order to promote our sport to a higher level, stronger, better direction.
\end{abstract}

\section{Introduction}

With the continuous development of sports, sports is showing the current modern competitive characteristics, and the level of competition is also rising, which the quantitative physical training, targeted and high load of a higher Claim. Coaches need long-term training information and a large amount of data accumulated by the athletes, while continuing research and practice, in order to gradually find some inherent law contained sports training, so as to continuously adjust the training methods and training policy [1]. A good sport coaches need to have a strong exploration and practicality, in addition to having a wealth of coaching experience, we also need to gradually master modern technological means to conduct scientific and rational sports training, so that athletes achieve better competition results.

\section{The Specific Application of Computer Technology in Physical Training}

\section{A. Establish the Appropriate Training Information Repository}

Computer technology applied to sports training among athletes training can collect information, organize, and analyze in order to establish the appropriate athlete management information system. Coaches can be adapted to different athletes to develop training plans based computer training data, optimize the appropriate training content. Computer-related data were collected athletes, thus a variety of training information in the form of files stored in the computer, and then the establishment of a corresponding training repository [2]. Coaches need to adjust based on the realities of the relevant documents, according to the corresponding data and then to develop a reasonable scientific training programs, so that the athletes can within a limited time, maximize and optimize the training effect. 


\section{B. Reasonable Adjustments Sports Training Strategies}

Application of computer technology in sports training, allows coaches to players based on the actual situation rationally adjust the corresponding sports training strategy. Coaches of computer technology in the simulation system to grasp the physical condition and the training of athletes, timely corresponding summary, to develop suitable for the athletes' physical fitness exercise stress analysis by the computer system, so that athletes after a reasonable physical exertion after that, to achieve the best physical recovery effect, thus effectively improve the quality of training [3]. In addition, coaches can also use computer technology to determine the athlete's strength, speed, load and body flexibility, quantitative evaluation of training effect and the physical condition of athletes, and then to develop a reasonable training program.

\section{Found the Deficiencies in Training}

The application of computer technology to sports training among sports training methods can be gradually from the traditional type of exploration experience gradual transformation to quantitative science, greatly enhancing the maturity of the athletes. Coaches in the control of training load of athletes, cannot rely on their own subjective feelings to assumptions, but needs accurate data as judged by the support, so that strength training athletes, the training cycle has become more rationalized [4]. Coaches Players accuracy of judgment, nor rely on the naked eye to observe, but need to use the computer and the coordination of a comprehensive analysis of high-speed cameras to motion picture player, and then found some flaws in the presence of athletes during training and disadvantages place. Players in the training process may be father, like some of the problems cannot be aware of their presence in the technical movements, coaches with the naked eye cannot effectively do the bystander, which requires a computer system to quantify the diagnostic Athletes action still exist which are not standard The place.

\section{Application of Computer Technology in Sport Competition}

\section{A. The Results of the Measurement Competition}

In sports, they usually involve the collection and comparison of data of Athletes, precision here the most critical is the data information. Like a swimming race, a swimmer to touch the end of the pool is to finish the race, the computer system may be a touch signal transport athletes to the end of the pool to the corresponding database among the precise time to record the extent of milliseconds, thus an important basis for athletes rankings. In tennis and badminton competitions, computer technology radar can spot hitting record and display the speed of athletes, "Eagle Eye" you can always visit football and tennis in the corresponding three-dimensional space trajectories and accurate landing site [5]. In addition, the football match in one electronic record instrument can effectively record a shot and the specific location of the next athlete shot, timely presented to the judge. Marathon runners need to install a small part in the sole, thus ensuring complete process run. Electronic sensing device is set on the ground receive relevant signals emitted by widgets, which are kept informed of the specific position of the players. These advanced techniques are based on the basis of modern computer technology applications which extend out of the application of computer-implemented computer graphics, you can simulate the corresponding stadium, utilizing three-dimensional forms, and thus simulate observed. Viewers can according to their actual needs to watch a different angle and position to achieve the perfect presentation of movement.

\section{B. Live Race}

In some integrated big game, the computer technology is playing an increasingly important role. 
In general, in order to allow space outside audience through television and other media to watch sporting events, it is necessary to use computer technology for data transmission and data transmission, and thus converted to the corresponding video information, showing off in front of an audience. In addition, computer technology can also live sporting events and sports-related rules, thus contributing commentator site in time for the audience and introduce the relevant competition process analysis, simultaneous transmission of information to the comments around the world. The advantage of this to get in the ball game in a vivid interpretation, for example, a basketball game site information into the system is usually caused by the system screen display system, arbitration video, time scoring system and other types of organic composition, these systems can achieve comprehensive information on data processing, which will be in the form of video-related information presented in front of an audience. Like our Beijing Olympic Games, it contains a number of venues, computer game technology can be carried out in each venue clearly presented in front of an audience, the audience according to their own preferences to choose the right sport to watch. Let the world are able to share sports events at the same time to celebrate this joyous occasion.

\section{Weather Prediction of Game}

Before sports, sports person in charge needs to grasp the weather venue, the Beijing Olympics used the Beijing Meteorological Bureau to provide IBM with accurate weather forecasting supercomputer. This computer can predict $44000 \mathrm{kmz}$ hourly weather data, weather forecast system than in the past with a more obvious function. This computer-based technology that extends out the weather forecast system can not only predict the weather conditions, but also can effectively monitor air quality, thus ensuring the smooth development of the Olympic Games. If we Olympics as a person, then the computer system is the nervous system, and, with the development of modern sports competition, this nervous system is playing an increasingly important practical role. The Olympic Games are the world's largest and most participatory sporting events, it has a complex engineering, data and other great features, the application of computer technology to meet the reasonable demands of the game to real information, meet the viewing needs of the audience outside, promote the smooth progress of the game sports.

\section{Conclusion}

In short, with the continuous development of computer technology, its application in sports training and sports is more widely. If the current sports training and physical lack of computer technology, then it cannot achieve the normal development of sports training and cannot guarantee the smooth implementation of the sports competitions. Computer technology being applied to sports, not only meet the needs of the athletes, but also to achieve the requirements of the viewing audience. Based on the arrival of the information age, contact sports and computer technology increasingly close. Organic integration of computer technology and sports, it is the future direction of the sports world.

\section{REFERENCE:}

[1] Bai Haijun, Gao Yun Lai Computer Application Virtual Reality Technology in College Physical Training [J] Heilongjiang Bayi Agricultural University, 2013,03: 105-107.

[2] Tao Modern Sports Training Computer Virtual Technology to explore [J] electronic test, 2013,11: 264-265. 
[3] Wang Jianhui, Yang Jun, Sun Lixin computer "virtual reality" technology in College Physical Training [J] North China Institute of Aerospace Engineering, 2012,02: 56-59.

[4] Sun Meng Discussion computer "virtual reality" technology in College Physical Training Application [J] electronic test, 2013,22: 61-62.

[5] Hong Xiu rocks on Competitive Sports application of computer technology [J]. Automation and Instrumentation, 2015,10: 142-143. 